\title{
¿Cómo va minando el entorno petrolero internacional a la economía mexicana?
}

\author{
How the international oil market weakens the Mexican economy?
}

\author{
Roberto Gutiérrez R.*
}

Resumen

Se analiza el impacto de la caída de los ingresos petroleros en la economía mexicana durante 2015-2016, provocado por la reducción de precios internacionales del petróleo, en dos ámbitos: la balanza de pagos y las finanzas públicas. Dado que el descenso ha sobrevenido en un periodo en que el comercio internacional crece a tasas lentas y México depende excesivamente de éste, así como de la captación de capitales, el efecto en la balanza de pagos es considerable. De la misma manera, las finanzas públicas han sufrido la reducción de la participación de los gravámenes a la industria petrolera en los ingresos tributarios de $33 \%$ hasta 2014 a $18 \%$ a partir de 2015 , $\sin$ que la reforma hacendaria se encuentre posibilitada para resarcir completamente esos recursos. Por tanto, no sólo se ha incrementado la participación de los Requerimientos Financieros del Sector Público (RFSP) en el Producto Interno Bruto (PIB), sino que en tan sólo tres años de su administración, el presidente Peña ha aumentado en 10 puntos porcentuales el saldo de la deuda del sector público respecto al PIB. Ante estas condiciones, las presiones de corto y mediano plazos sobre la economía mexicana crecen, máxime si se tiene en cuenta que los ahora exiguos ingresos esperados de inversión extranjera directa (IED) asociados a la reforma energética se aplazarán al menos hasta 2017, y el aterrizaje de la política monetaria impulsado por la Reserva Federal (FED) de Estados Unidos (EU), que por lo menos implicará tres aumentos de la tasa de interés de referencia en aquel país durante 2016, producirá aumentos iguales o mayores en la de México. Esto encarecerá el costo no sólo de la deuda pública, sino también de la privada, que en los años recientes ha crecido a tasas inclusive más altas a las del sector público.

\footnotetext{
Palabras clave:

- Petróleo

- Precios

- Balanza de pagos

- Finanzas públicas

- Crecimiento
}

\begin{abstract}
The impact of Mexico's oil revenues fall during 2015-2016 due to lower international oil prices is analyzed, observing that it affects two areas: the balance of payments and the public finances. Given the fact that the decline has occurred in a period in which international trade grows at slower rates, and Mexico relies too heavily on it, as well as on the inflow of international capitals, the effect on the balance of payments is considerable. Similarly, public finances have suffered the reduction of oil industry taxes participation on total tax revenues from 33\% up to 2014 to $18 \%$ in 2015-2016, being not the tax reform fully capable to reimburse the government such resources. Therefore, not only the participation of the Public Sector Borrowing Requirement (PSBR) in the Gross Domestic Product (GDP) has grown, but in just three years in office, the Peña administration has increased 10 percentage points the public sector debt in terms of GDP. Under these conditions, the pressures of short and medium term on the Mexican economy increases, especially if one considers that expected Foreign Direct Investment (FDI) revenues associated to the energy reform will be postponed and substantially reduced, at least until 2017, and the landing of Federal Reserve's (FED) monetary policy, which will involve at least three increases in the reference interest rate of the United States (US) during 2016, will produce equal or greater increases in Mexico's. This will not only impact the cost of public but also private debt, which disbursement in recent years has grown even faster than that of the public sector.
\end{abstract}

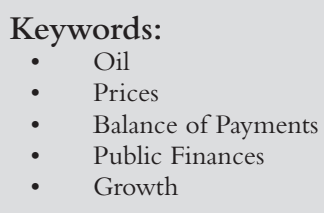

JEL: L71, E62, O11 


\section{El problema}

En los 35 años transcurridos desde que México volvió a ser una potencia petrolera, alcanzando en 2004, punto más alto de la producción del yacimiento Cantarell, un nivel de 3.38 millones de barriles diarios (BD), con exportaciones de 1.87 millones de $\mathrm{BD}$, el país ha enfrentado cinco recesiones (gráfica 1). ${ }^{1}$ Las dos primeras (1982-83 y 1986) estuvieron estrechamente vinculadas a la caída del precio internacional del petróleo. La de 1995 tuvo que ver con un factor eminentemente interno, el alto nivel de endeudamiento de los sectores público y privado, combinado con fallas elementales de regulación de un el sistema bancario recién reprivatizado. Las de 2001 y 2009 vinieron de EU; la primera vinculada al sobrecalentamiento de la industria de Tecnologías de la Información y la Comunicación (TIC), cuyo desinflamiento arrastró a los mercados financieros de todo el mundo, y la segunda a una crisis originada por la liberalización del sistema financiero, que se agravó por el inadecuado manejo de los créditos hipotecarios y al consumo privado, irradiándose inmediatamente al ámbito internacional.

Aunque en las dos últimas recesiones se observó también un debilitamiento de los precios internacionales del petróleo -en 2001 cayeron en promedio 14\% respecto al año anterior y en 2009 bajaron 63\% (BP, 2015) - estos movimientos se dieron como consecuencia de la desaceleración económica mundial y no de un incremento inusual de la oferta de crudo, por lo que su recuperación fue rápida. Mientras tanto, México tenía a su favor tres puntos: i) el saldo del endeudamiento externo del sector público se mantenía relativamente controlado (32.3\% respecto al PIB en 2001 y $33.1 \%$ en 2009); las finanzas públicas exhibían un nivel de déficit bajo en términos de RFSP, sobre todo el de 2001, 2.7\% (el de 2009 subió medio punto para apoyar la actividad económica y 2 puntos más en virtud de que la reforma energética de 2008 implicó descontar a Pemex de su base gravable los gastos de inversión) (SHCP, 2015a), y el saldo global de la balanza de pagos - suma del saldo de la cuenta corriente y la cuenta de capital- mantuvo un nivel de superávit relativamente alto: 10731 miles de millones de dólares en 2001 y 9058 miles de millones en 2009 (Banxico, 2015).

\footnotetext{
${ }^{1}$ México fue por primera vez potencia petrolera entre 1920 y 1925, con una producción promedio anual de 427 mil BD, de la que más de $90 \%$ era exportada por las empresas petroleras internacionales, como se deduce de Meyer (1994: 236).
} 


\section{Gráfica I}

Variación anual del PIB de México, 1980-2015

Base 2008 $=100$

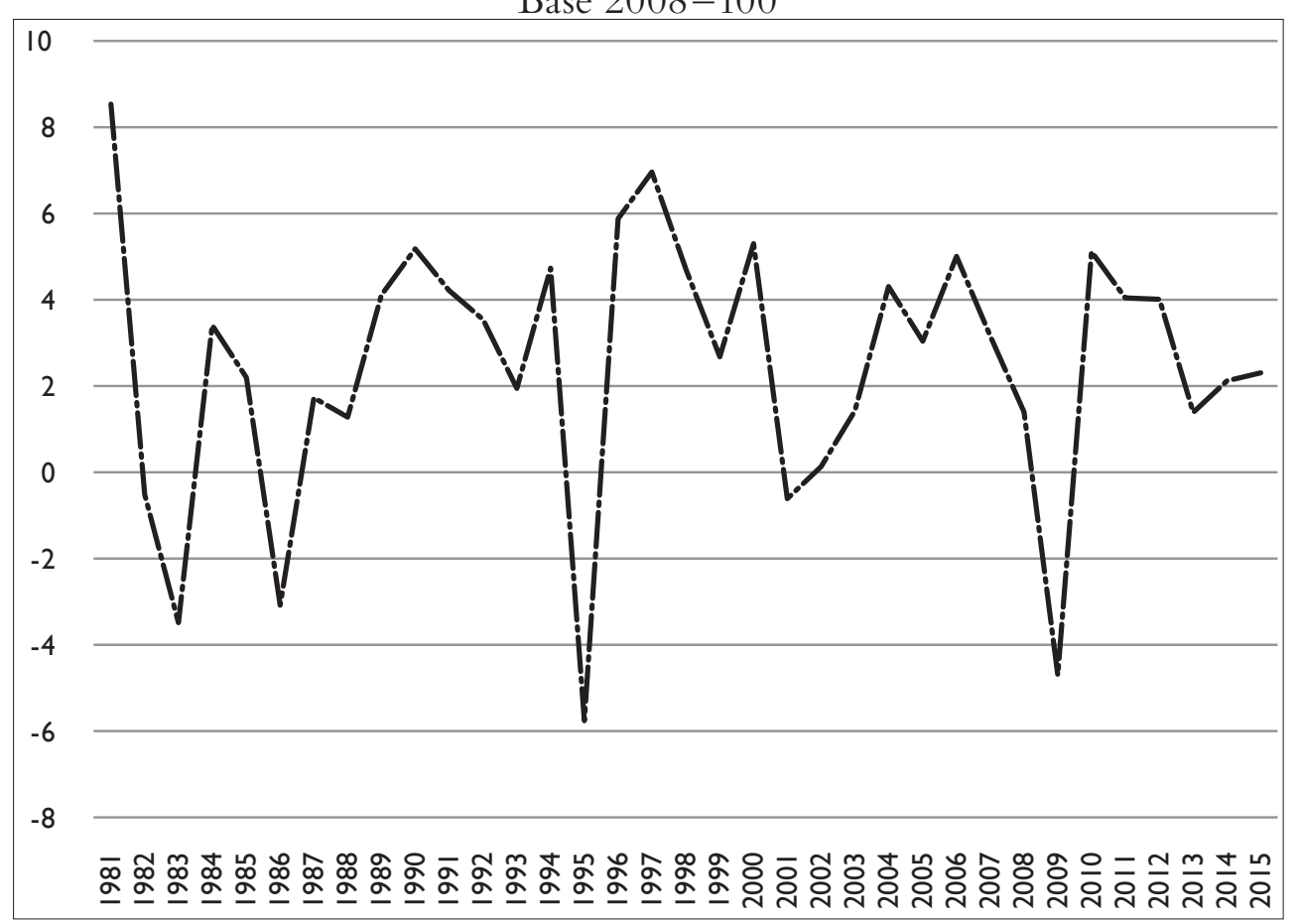

Fuente: con base en datos del INEGI (2015).

En 2015-2016 las condiciones son diferentes: el saldo de la deuda del sector público representa 47\% del PIB (tan solo durante los tres primeros años de la administración Peña ha subido 10 puntos porcentuales); los RFSP ascienden a 4.1\% del PIB (SHCP, 2015b) y la balanza global tiene un saldo cercano al déficit (Banxico, 2015).

\section{Vinculaciones macroeconómicas}

Evidentemente el desempeño de la industria petrolera se liga por diversas vías a los acontecimientos anteriores. Por el lado de la cuenta corriente, a través del menor ingreso de divisas, que ha provocado por primera vez desde mediados de los años setenta del siglo xx un déficit de la balanza petrolera de Pemex (Pemex, 2015), el cual tiende a aumentar en 2016. Además, como muestra la gráfica 2, la pérdida de ingresos petroleros, junto con la necesidad de apoyar el tipo de cambio, provocó una disminución sostenida de las reservas inter- 
nacionales en poder del Banco de México (Banxico) durante prácticamente todo 2015, situación que seguramente se mantendrá al menos en una parte de 2016. Sobre este punto debe tenerse presente el hecho de que, históricamente, $88 \%$ del total de divisas que Pemex recibe pasan al Banxico en la forma de reservas (Heath, 2012: 215).

\section{Gráfica 2}

\section{Relación entre las reservas internacionales de Banxico}

(eje izquierdo, línea continua en miles de millones de dólares)

\section{y el precio de la mezcla mexicana de exportación}

(eje izquierdo en barras sólidas en dólares/barril)

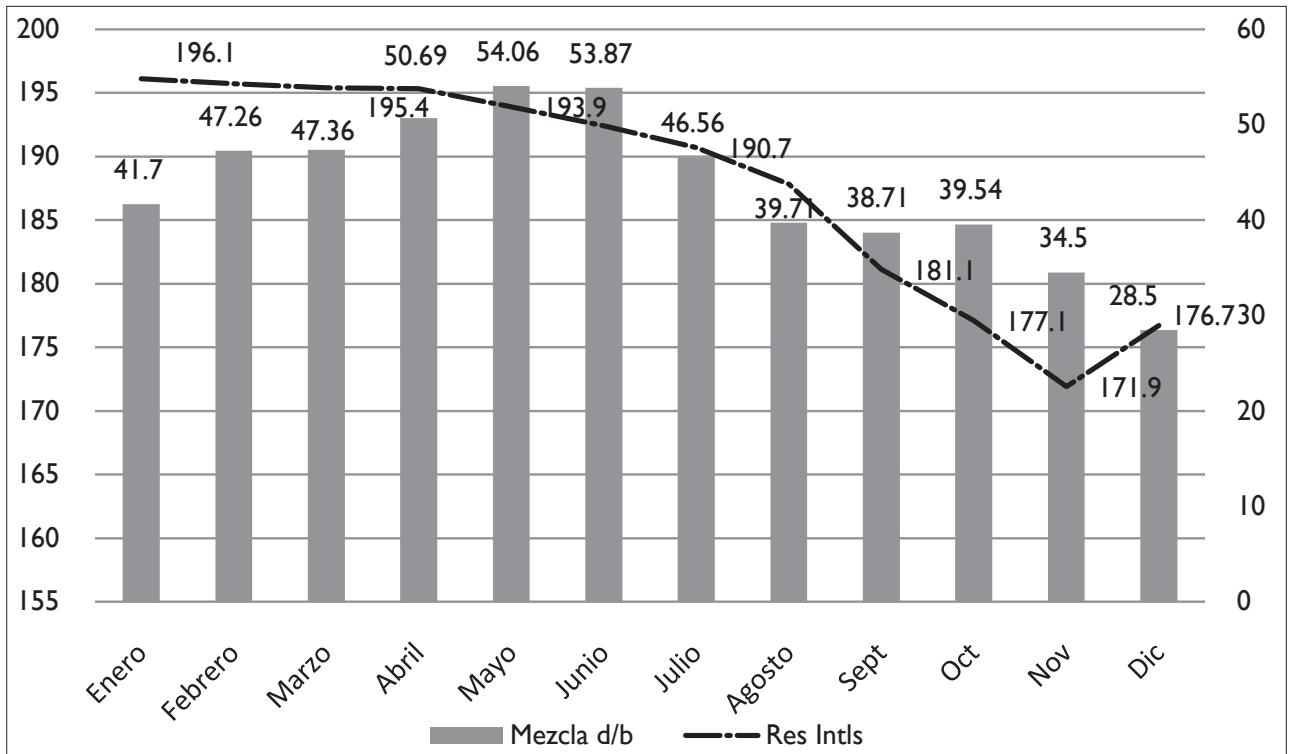

Fuentes: Pemex (2015) y Banxico (2015).

Por el lado de los RFSP y el saldo de la deuda del sector público, ambos han tendido a aumentar en 2015-2016 debido a que la contribución de Pemex a los ingresos tributarios bajó de 33\% en los años previos a la caída de los precios del petróleo a apenas 18\% en 2015-2016, y los mayores ingresos tributarios obtenidos por otros gravámenes gracias a la reforma hacendaria de 2013 (poco más de dos puntos porcentuales adicionales con relación al PIB en 2014-2015) no han sido suficientes para paliar la pérdida por recaudación a Pemex, incluyendo condonaciones de impuestos dada la precariedad de su situación. 
En términos del PIB, la pérdida anterior asciende a un poco más de tres puntos porcentuales: en 2013 los ingresos presupuestarios de origen petrolero ascendían a casi 7.5\%, y en 2016 llegarán a apenas 4.2\%. Como contraparti$\mathrm{da}$, los ingresos presupuestarios de origen no petrolero pasan de $10.5 \%$ en el primer año a $12.7 \%$ en 2016 (SHCP, 2015b: 83). Esto quiere decir que el total de ingresos presupuestarios respecto al PIB bajó de 18 a 16.9\%, una pérdida de 1.1 puntos porcentuales.

El precio internacional del crudo atraviesa en enero de 2016 por su nivel más bajo desde 2003, menos de 25 dólares por barril-d/b, y el tipo de cambio se ha depreciado $40 \%$ a partir del nivel de resistencia de 13 pesos por dólar ( $\mathrm{p} / \mathrm{d})$ que sostuvo hasta la primera parte de 2014; ambas tendencias muestran una estrecha correlación, como evidencia la gráfica 3.

\section{Gráfica 3}

\section{Precio de la mezcla mexicana de exportación}

(eje izquierdo en $\mathrm{d} / \mathrm{b}$ )

\section{y tipo de cambio peso/dólar}

(eje derecho, línea discontinua) en 2015

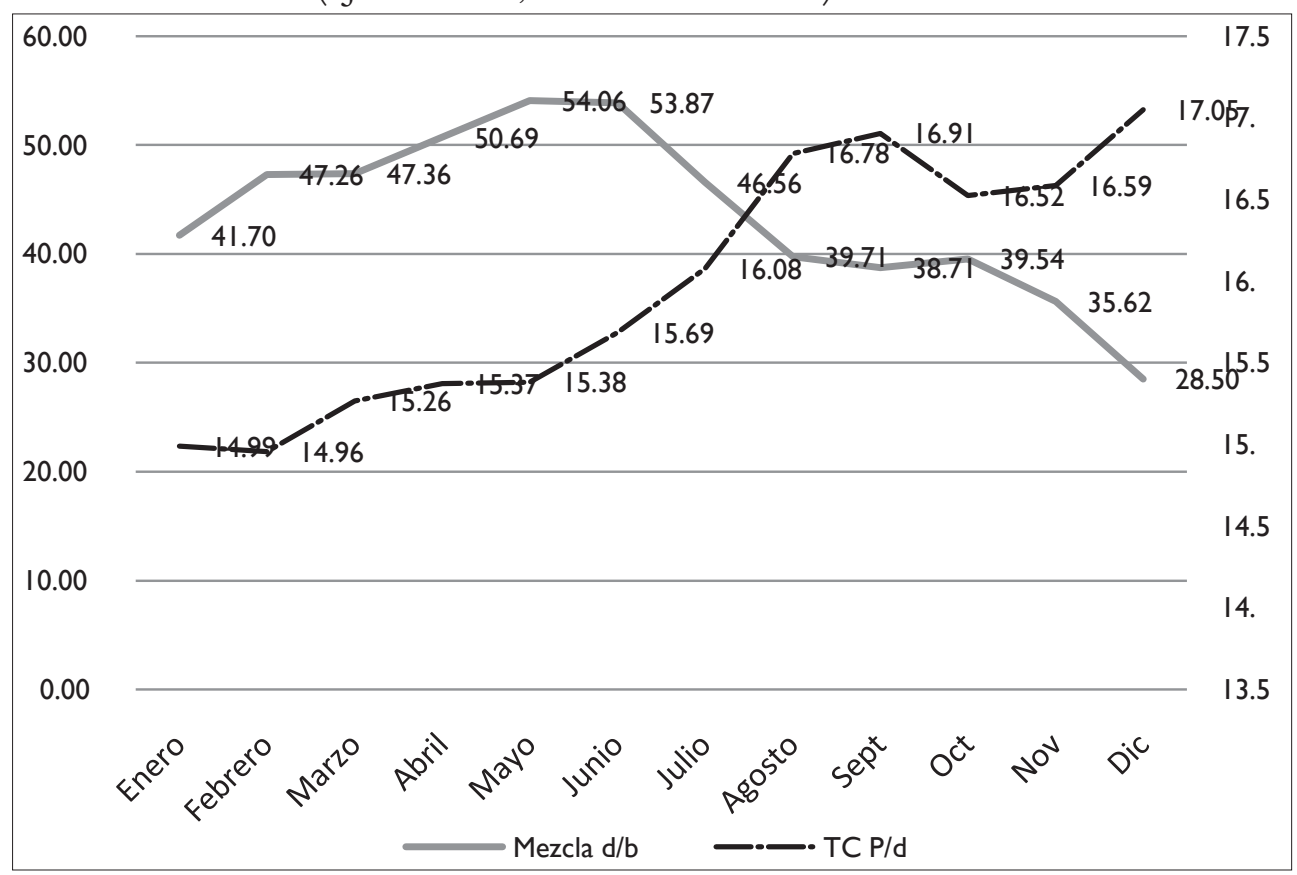

Fuentes: con base en Pemex (2016) y Banxico (2015). 
Aun con todo esto, y a pesar del hecho de que en América Latina dos de las seis economías más grandes, Brasil y Venezuela, pasan en 2015-2016 por una profunda recesión y se espera que el crecimiento de la región en 2016 sea de cero, la economía mexicana mantiene la perspectiva de crecer por lo menos a una tasa de $2.3 \%$. Esto se explica porque a lo largo de los años se ha ido reduciendo el peso de la industria petrolera en la generación de divisas, de manera que hoy día 85\% de las exportaciones de mercancías corresponden a la industria manufacturera. Esta decreciente dependencia en un commodity muy volátil ha contribuido, junto con las remesas familiares, a reducir el efecto de la caída de su valoración internacional en la cuenta corriente de la balanza de pagos. No sucede lo mismo con la cuenta de capital y financiera, que depende de la percepción que en el exterior se tiene de la economía mexicana, así como del arbitraje de las tasas de interés, que en los dos últimos años dejó de ser favorable para México, habida cuenta de que el diferencial respecto a EU es de menos de tres puntos porcentuales (menos de 300 puntos base), y eso no ha alcanzado para cubrir el riesgo cambiario.

\section{Consecuencias}

Los bajos gravámenes a la industria en general, y en particular a las ramas manufactureras que son promocionadas por el gobierno federal (programas ALTEX, ECEX, PROSEC, IMMEX, etc. Economía, 2015), han hecho recaer el más alto peso de la carga tributaria en dos tipos de contribuyentes, Petróleos Mexicanos (Pemex) y la fuerza de trabajo. Con el paso de los años, esto ha conducido a dos problemas. Por una parte, al debilitamiento de Pemex, hasta ponerlo al borde de la inoperancia, ya que ni siquiera puede cumplir con las asignaciones de la Ronda Cero y tiene que buscar alianzas para mantenerse en el negocio. Por otra, a la depauperación de las remuneraciones al sector formal de la economía (el informal mejor no se considera), lo que ha llevado a que la participación de dichas remuneraciones en el PIB se ubique a partir de 2013 en su nivel más bajo casi desde que se dispone de registros, 27 por ciento.

Como sostiene Romero Tellaeche (2014), desde la anexión del país al Tratado de Libre Comercio de América del Norte (TLCAN) el gobierno mexicano se encuentra maniatado en términos de política económica. Además, desde el punto de vista de la caída de los ingresos, poco ayudan las coberturas petroleras: en 2015 se perdieron 15363 millones de dólares, si se tiene en cuenta que por cada dólar que baja el precio de la mezcla mexicana el país deja de recibir 434 mil dólares al año, y la cifra presupuestada fue de $35.4 \mathrm{~d} / \mathrm{b}(79 \mathrm{~d} / \mathrm{b}-43.6 \mathrm{~d} / \mathrm{b})$. 
Si se consideran los cálculos que se hicieron para recibir las coberturas petroleras (se protegieron 228 millones de barriles de crudo a un precio de $76.4 \mathrm{~d} / \mathrm{b})$, la cantidad recibida debió haber sido de 7478 millones de dólares (Gutiérrez R., 2015) pero la Secretaría de Hacienda y Crédito Público (SHCP) anunció a fines de 2015 que se le entregarían 6200 millones de dólares. Una pequeña parte de la diferencia puede ser porque el periodo cubierto no coincide exactamente con el año calendario; el resto es algo que tal vez quiera aclarar la SHCP. Debe resaltarse que si al primer cálculo se agregan los dólares destinados a la compra de coberturas, la pérdida de divisas se ubica en 16136 millones de dólares, y eso representa casi 1.4\% del PIB.

Para 2016 la pérdida respecto a 2014 va a ser aún más alta, ya que el precio promedio de la mezcla mexicana de exportación podría situarse en $25 \mathrm{~d} / \mathrm{b}$, lo que implica un diferencial de $54 \mathrm{~d} / \mathrm{b}$ y por tanto ingresos no recibidos de 23,436 millones de dólares. Si se toma como referencia la cifra presupuestada de $50 \mathrm{~d} / \mathrm{b}$ para 2016, la pérdida se ubica en 10850 millones de dólares, y si se considera lo que podría recibirse en términos netos gracias a las coberturas, los cálculos serían como siguen: se aseguraron 212 millones de barriles a un precio de $49 \mathrm{~d} / \mathrm{b}$. Por tanto, podrían recuperarse 5088 millones de dólares. Al considerar el costo de la prima, que fue de 1090 millones de dólares, la pérdida acumulada de recursos respecto a lo que se habría recibido si las cosas se mantuvieran como hasta 2014 sería de 24526 millones de dólares, y respecto a 2015 de 6178 millones de dólares. Empero, lo que se recuperaría en términos netos gracias a las coberturas serían 3998 millones de dólares.

\section{La balanza de pagos}

El problema con la pérdida de divisas por la reducción del precio internacional del petróleo es que no existen posibilidades de suplir esos recursos mediante una o varias de las cinco principales fuentes de captación de divisas que registra la balanza de pagos, como se explica a continuación, con base en Banxico (2015).

1. La inversión extranjera total se redujo -14 476 millones de dólares entre enero y septiembre de 2015 respecto a igual periodo de 2014, ya que

a. aunque la IED aumentó 3340 millones,

b. la inversión de cartera disminuyó -14 476 millones.

2. La balanza de productos manufacturados tuvo durante los 10 primeros meses del año un déficit de -8364 millones de dólares.

3. En igual periodo, la balanza de productos petroleros (Pemex y compañías 
privadas) acusó en igual lapso un déficit de -7 648 millones de dólares.

4. Las remesas familiares ascienden en el año completo, de acuerdo con cifras preliminares, a un poco más de 25000 millones de dólares.

5. Y el saldo por turismo internacional entre enero y octubre fue de 5939 millones de dólares.

Sumando con el signo correspondiente los saldos anteriores para obtener una idea de magnitud anual, se obtienen -451 millones de dólares. Debe resaltarse que esas cincos cuentas (seis si se separa a la inversión IED e inversión en cartera) representan 98\% de los ingresos de divisas de la balanza de pagos (Gutiérrez, 2011: 136). Así que en 2015-2016 las presiones que resiente la economía se dan no sólo en la cuenta corriente, sino también en la cuenta de capital y financiera, y esa es una situación que no se vivía desde 2009.

\section{Conclusiones}

Se observa un incremento inusitado en las presiones de corto y mediano plazo de la economía mexicana en virtud de que la reducción de precios de su principal materia prima de exportación y hasta hace año y medio fuente insustituible de los ingresos tributarios del gobierno federal, ha experimentado una reducción de más de $75 \%$ entre junio de 2014 y enero de 2016 . A esto se agregan cuatro problemas fundamentales.

En primer lugar, los ingresos realmente significativos de IED dirigidos a la industria petrolera se aplazarán al menos hasta 2017 (siempre y cuano las empresas petroleras internacionales se deciden a ingresar al negocio del mar profundo del Golfo de México), y no sería realista esperar que el país recupere en 2016 la capacidad de captar inversión de cartera como lo hizo entre 2010 y la primera mitad de 2014, sobre todo porque ha habido una reorientación en el destino de los capitales de las economías emergentes a las desarrolladas.

En segundo lugar, el aterrizaje de la política monetaria de la FED a partir de diciembre de 2015, que se traduce en por lo menos tres aumentos de la tasa de interés de referencia en aquel país durante 2016, conducirá a aumentos iguales o mayores en la de México, lo que encarecerá el costo no sólo de la deuda pública, sino también de la privada, que en los años recientes ha crecido a tasas inclusive más altas a las del sector público.

En tercer lugar, la economía mundial continuará creciendo a tasas bajas, que como nunca desde la postguerra se están traduciendo en muy lentas tasas 
de crecimiento del comercio mundial, y es precisamente en éste el que México busca sustentar su crecimiento económico.

En cuarto lugar, y dadas las experiencias de los años ochenta y noventa, en que la caída de los precios del petróleo no condujo sólo a una sino a varias reducciones del gasto presupuestal en un mismo año, existe la posibilidad de que los dos recortes anuales observados recientemente, uno en enero de 2015 y otro al preparar el presupuesto base cero para 2016, puedan repetirse en este segundo año, con las implicaciones que ello tendría para el crecimiento.

Así que las previsiones del gobierno mexicano y de los organismos internacionales de que en 2016 se acelerará un poco el crecimiento observado en 2015 -situado en el nivel prácticamente de reposición de los últimos 23 años- parecen muy difíciles de cumplirse. Lo más importante es dar los pasos adecuados para evitar que el elevado endeudamiento de los sectores público y privado y los mayores niveles del servicio de la deuda se conviertan en un obstáculo para hacer efectiva la recuperación en 2017.

\section{Referencias bibliográficas}

Banco de México (Banxico) (2015), Balanza de pagos, www.banxico.org.mx consultado en enero de 2016.

British Petroleum (BP) (2015), Statistical Review of World Energy 2015, www.bp.com consultado en enero de 2016.

Economía (Secretaría de Economía) (2015), "Instrumentos de comercio exterior", en www.economia.gob.mx consultado en diciembre de 2015.

Gutiérrez R., Roberto (2015), "Efectos del choque petrolero de 2014-2015 en la captación de divisas y el gasto público", Economía Informa núm. 391, marzo-abril.

__ (2011), "El sector exportador y la balanza de pagos de México después de la recesión económica de 2008-2009", Denarius núm. 22, marzo.

Heath, Jonathan (2012), Lo que Indican los Indicadores, México: INEGI.

Instituto Nacional de Estadística y Geografía (INEGI) (2015), Base de datos, en www. inegi.org.mx consultado en enero de 2016.

Meyer, Lorenzo (1994), "El desarrollo de la industria petrolera en México", en Enrique Cárdenas (compilador), Historia Económica de México, Lecturas de El Trimestre Económico, vol. IV, México: FCE.

Romero Tellaeche, José (2014), Los límites al crecimiento económico de México, México: Colmex y UNAM.

Secretaría de Hacienda y Crédito Público (SHCP) (2015a), Estadísticas presupuestales, en www.shcp.gob.mx consultado en diciembre de 2015. consultado en diciembre de 2015. 